\title{
Girls with a History of Premature Adrenarche Have Advanced Growth and Pubertal Development at the Age of 12 Years
}

\author{
Jani Liimatta*, Pauliina Utriainen, Raimo Voutilainen and Jarmo Jääskeläinen \\ Department of Pediatrics, University of Eastern Finland and Kuopio University Hospital, Kuopio, Finland
}

Background: Premature adrenarche (PA) has been linked to early thelarche and menarche, but longitudinal data on growth and pubertal development after PA are insufficient.

Methods: Growth and pubertal development of mostly full-term and appropriate for gestational age-born 43 PA (36 girls) and 63 control children (52 girls) were analyzed prospectively. Children examined first at the mean age of 7.6 years were reexamined at

OPEN ACCESS

Edited by:

Mohamad Maghnie,

Università di Genova, Italy

Reviewed by:

Laura Audí,

Vall d'Hebron Research

Institute, Spain

Hugo Fideleff,

Universidad de Buenos

Aires, Argentina

Luigi R. Garibaldi,

University of Pittsburgh-

Children's Hospital, United States

*Correspondence:

Jani Liimatta

jani.liimatta@kuh.fi

Specialty section: This article was submitted to Pediatric Endocrinology, a section of the journal Frontiers in Endocrinology

Received: 25 August 2017 Accepted: 12 October 2017 Published: 31 October 2017

Citation:

Liimatta J, Utriainen $P$, Voutilainen $R$ and Jääskeläinen J (2017) Girls with a History of Premature Adrenarche Have Advanced Growth and Pubertal Development at the Age of 12 Years.

Front. Endocrinol. 8:291. doi: 10.3389/fendo.2017.00291 the mean age of 12.0 years.

Results: The PA girls but not the boys were taller and had higher body mass index (BMI) than the controls. A higher proportion of the PA than control girls had reached menarche, while the same percentage of the PA and control boys were at Tanner genital stage $\geq 2$. The PA girls with premature pubarche (PP) were taller but not heavier and had more often reached menarche by the age of 12 years than the PA girls without PP. The PA girls with menarche had lower birth length $(\mathrm{BL})$ and higher prepubertal insulin-like growth factor 1 (IGF-1) concentrations compared with non-menarcheal PA girls. In logistic regression analyses for all girls, lower BL standard deviation score, earlier maternal menarche, and higher prepubertal IGF-1 were independently associated with menarche.

Conclusion: At 12 years of age, the PA girls had higher BMI, advanced linear growth, and accelerated pubertal development with earlier menarche than the control girls. The PA girls with PP were taller and had earlier menarche than the PA girls without PP. Lower $\mathrm{BL}$ and higher prepubertal IGF-1 concentration were predictive factors for menarche by the age of 12 years.

Keywords: premature adrenarche, dehydroepiandrosterone sulfate, insulin-like growth factor I, growth, puberty, menarche

\section{INTRODUCTION}

In adrenarche, developing adrenocortical zona reticularis produces increasing amounts of adrenal androgen precursors, mostly dehydroepiandrosterone sulfate (DHEAS). Increasing androgenic activity leads to clinical signs: adult-type body odor, oily hair and skin, comedones/acne, and axillary and pubic hair. If the clinical signs, together with elevated DHEAS concentrations, are seen before the

Abbreviations: AGA, appropriate for gestational age; BL, birth length; BMI, body mass index; cMPH, corrected mid-parental height; DHEAS, dehydroepiandrosterone sulfate; IGF-1, insulin-like growth factor 1; PA, premature adrenarche; PP, premature pubarche; SGA, small for gestational age; SDS, standard deviation score. 
age of 8 years in a girl or 9 years in a boy, adrenarche is regarded as premature [reviewed in Ref. $(1,2)]$. In Finland, the prevalence of premature adrenarche (PA) is $8.6 \%$ in girls and $1.8 \%$ in boys (3). Despite a relatively high prevalence, the long-term outcome of PA is still poorly known.

In several studies, PA has been linked to unfavorable metabolic features including obesity $(4,5)$ and hyperinsulinism or insulin resistance (6-8). Low birth weight or being born small for gestational age (SGA) has been associated with PA in retrospective studies from Australia, Brazil, and Spain $(5,9,10)$. Advanced prepubertal growth in height has been reported in most PA cohort studies (11-14). One possible link between accelerated statural growth and PA is insulin-like growth factor 1 (IGF-1) (15) whose increased concentrations have been found in PA children $(14,16,17)$.

Only few PA cohorts have been followed intensively until adolescence or adulthood $(9,18)$. In these longitudinal studies, PA girls have had earlier timing of thelarche and menarche than their peers. Of note, a substantial percentage of the PA girls in these studies have been born SGA, which itself may lead to early catchup growth and earlier menarche (19). Earlier timing of menarche was seen also in one retrospective study (13). Although children with PA usually have advanced bone age $(11,17)$ and prepubertal statural growth (11-14), final height is mostly reported to be normal $(9,12,13,18)$.

At the baseline of our longitudinal follow-up study on PA, overweight, hyperinsulinism, decreased insulin sensitivity, and childhood metabolic syndrome were more common in the PA than control girls at the mean age of 7.6 years (6). The girls with PA were also taller than their peers with higher serum IGF-1 concentrations (14). In this follow-up study, we examined whether the anthropometric differences found in prepuberty persist until adolescence and if PA is associated with advanced pubertal development at 12 years of age. Finally, we explored which prepubertal factors predict earlier menarche in PA and healthy girls.

\section{SUBJECTS AND METHODS}

\section{Subjects and Design}

Our original cohort study on 73 Finnish children with PA and 99 age- and sex-matched healthy controls has been reported previously (20). We invited the children to attend a follow-up visit at the age of 12 years. Altogether 106 subjects participated (61.6\% of the original cohort; 36 PA, 52 control girls; 7 PA, 11 control boys), and they were all examined at the pediatric outpatient clinic of Kuopio University Hospital. The PA children had presented with at least one clinical sign of adrenal androgen action before the age of $8 / 9$ years (girls/boys, respectively) together with a serum DHEAS concentration over $1 \mu \mathrm{mol} / \mathrm{L}$ and other reasons of hyperandrogenism (including central puberty, congenital adrenal hyperplasia, androgen producing tumor) were excluded. Five children had been born SGA [birth weight $<-2$ standard deviation score (SDS); $4.7 \%$ overall; $1 / 36$ PA vs $3 / 52$ control girls; $1 / 7$ PA vs 0/11 control boys; both NS]. Five girls, but none of the boys had been born preterm (4.7\% overall; 1/36 PA vs 4/52 control girls; NS). The study protocol was approved by the Research
Ethics Committee of the Hospital District of Northern Savo, and an informed consent was obtained from the children and their parents in accordance with the ethical principles stated in the Declaration of Helsinki.

\section{Clinical Evaluation}

Gestational age, birth size, anthropometric measures, pubertal stage, and biochemical results at the mean age of 7.6 years had been recorded earlier. At the current 12-year-age follow-up visit, anthropometric measures, pubertal stage, and menarcheal timing were assessed. Bone age determination was not included in the study protocol for ethical reasons (i.e., radiation exposure). The parents were enquired upon their heights and timing of puberty (maternal age at menarche and paternal age during pubertal peak height velocity). These were subjective estimates given by the parents, but as both growth and puberty are systematically monitored in Finland until the end of secondary education, individuals are well aware of their final height and pubertal tempo. Paternal pubertal growth spurt was classified into three categories (early, on-time, and late) as compared with that of their peers: a difference more than a year was considered as early or late timing of the pubertal growth spurt.

Height was measured with a calibrated Harpenden stadiometer (Holtain Ltd., Crymych, UK) and recorded to the nearest $0.1 \mathrm{~cm}$ as the mean of three repeated measurements. Weight was measured with a calibrated electronic scale after an overnight fast and recorded to the nearest $0.1 \mathrm{~kg}$. Waist circumference was measured after expiration at mid-distance between the bottom of the rib cage and the top of the iliac crest. Body mass index (BMI) was calculated as the weight in kilograms divided by the square of height in meters. Birth length (BL), birth weight, height, and BMI SDS were calculated with current Finnish growth references $(21,22)$. Pubertal development was evaluated by a trained physician (P.U.) with Tanner staging scores $(23,24)$. Age of menarche was defined as precisely as possible by interviewing the girls and their parents. Corrected mid-parental height (cMPH) was calculated by the method of Tanner (25): mid-parental height $-6.5 \mathrm{~cm}$ for girls and mid-parental height $+6.5 \mathrm{~cm}$ for boys.

\section{Biochemical Analyses}

All biochemical samples had been drawn and analyzed at the baseline visit (mean age of 7.6 years) and the analytical methods have been described more closely in the previous reports $(6,14,20)$. The blood samples were drawn after an overnight fast and stored at $-80^{\circ} \mathrm{C}$ until assayed. Serum insulin concentrations were analyzed with a specific time-resolved fluoroimmunoassay by AutoDelfia (PerkinElmer Life and Analytical Sciences Wallac Oy, Turku, Finland), DHEAS, and androstenedione concentrations with specific Coat-A-Count RIAs (Diagnostic Products Corporation, Los Angeles, CA, USA), and IGF-1 concentration by an immunochemiluminometric assay using a Diagnostic Products IMMULITE 2000 analyzer.

\section{Statistical Analyses}

All statistical analyses were performed using the SPSS 23.0 software (IBM Corp., Armonk, NY, USA). The distributions for normality were first tested with the Shapiro-Wilk test and 
visually from the histograms. The independent samples $t$-test for normally distributed variables and the Mann-Whitney $U$ test for non-normally distributed variables were used to compare differences in the means and medians between the study groups, respectively. To evaluate statistical differences in pubertal development between the study groups in crosstab settings, the Pearson $\chi^{2}$ test or Fisher's exact test was used. Binary logistic regression analyses were used to evaluate predictive factors for menarche (categorical dependent variable: menarche, yes or no). Continuous non-normally distributed biochemical covariates in the regression analyses were first logarithmically transformed and then used as SDS. The associations or statistical differences with $P<0.05$ were considered significant.

\section{RESULTS}

Results were analyzed separately for females and males. Characteristics of the PA and control girls and boys at birth, at the age of 7 years (=baseline), and at the age of 12 years (=follow-up visit) are depicted in Table 1. Subgroup comparisons of the PA girls with $(\mathrm{PA}+\mathrm{PP})$ and without premature pubarche $(\mathrm{PA}-\mathrm{PP})$ at the baseline, and the PA girls with and without menarche at the age of 12 years are depicted in Table 2 .

\section{Anthropometry}

Gestational age, birth weight, and age at baseline or follow-up visit did not differ between the PA and control girls (Table 1). The PA girls participating in the current examination at the age of 12 years had significantly lower BL than the control girls, but the difference in BL SDS did not reach statistical significance (Table 1). At the follow-up visit, the girls with a history of PA were taller (height SDS 0.88 vs $-0.08, P<0.001$ ) and had higher BMI SDS (0.81 vs 0.13, $P=0.002$ ) than the control girls (Table 1). Moreover, the PA + PP girls were taller (height SDS 1.25 vs 0.44 , $P=0.041$ ) but not heavier than the PA - PP girls at the age of 12 years (Table 2). No statistically significant differences were detected in anthropometrics between the PA and control boys.

\section{Pubertal Development}

Pubertal development of the children with PA and controls is depicted in Figure $\mathbf{1}$ and Table 3. There were no significant differences in the percentage of children with ongoing puberty

TABLE 1 | Characteristics of children with PA and control children at birth, at the age of 7 years, and at the age of 12 years.

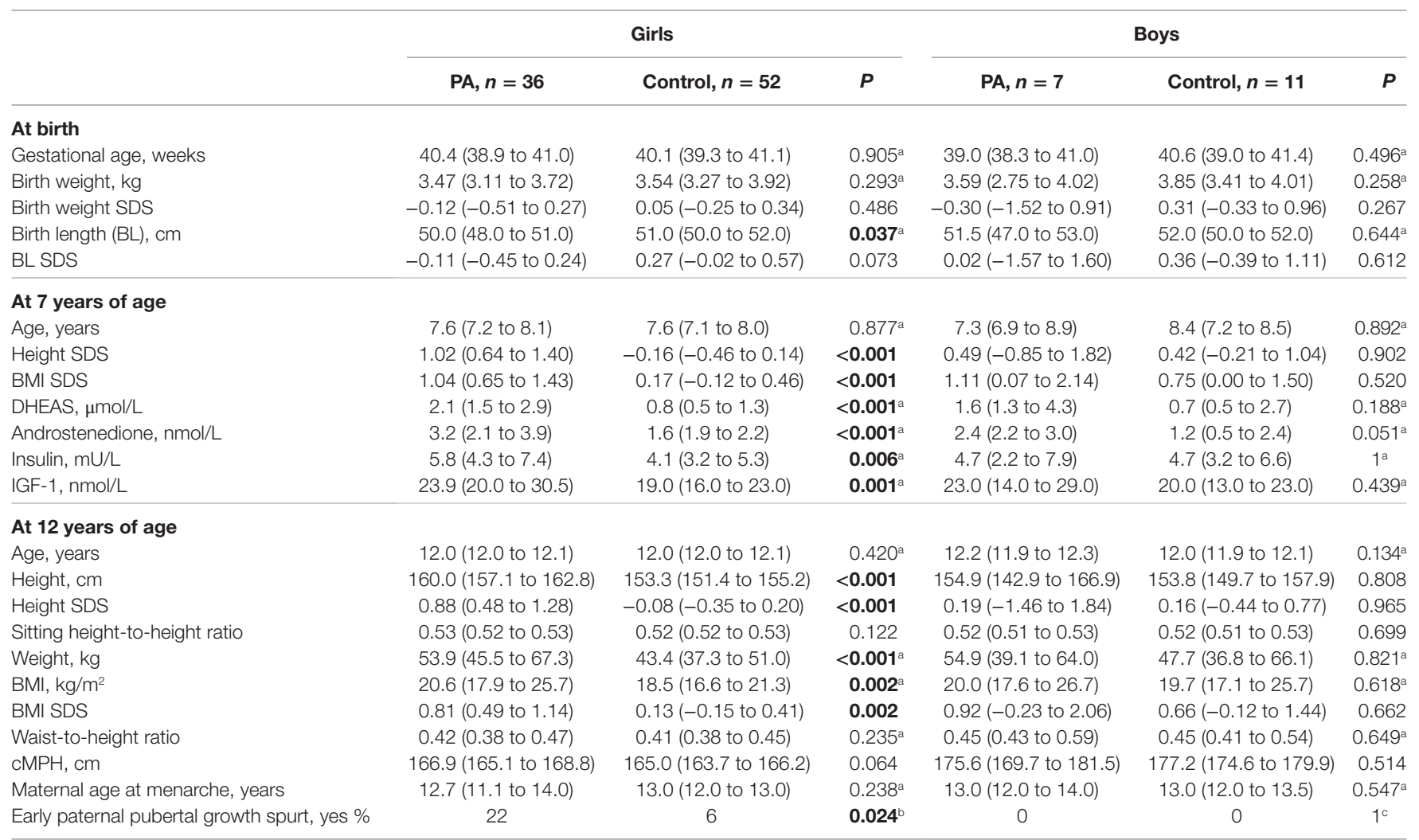

Values are expressed as mean (95\% confidence interval), and statistical differences between the study groups were analyzed

using independent samples $t$-test, unless noted otherwise. Significant p-values $(<0.05)$ are highlighted with bold.

aMedian (interquartile range) and Mann-Whitney $U$ test for non-normally distributed variables.

$b, c$ Percentage and Pearson $\chi^{2}$ test $^{b}$ or Fisher's exact test ${ }^{c}$ for categorical variable.

PA, premature adrenarche; SDS, standard deviation score; BMI, body mass index; DHEAS, dehydroepiandrosterone sulfate; IGF-1, insulin-like growth factor 1;

$\mathrm{CMPH}$, corrected mid-parental height. 
TABLE 2 | Comparisons of the follow-up data between the subgroups of the PA girls with (PA + PP) and without premature pubarche (PA - PP), and the PA girls with $(P A+M)$ and without menarche $(P A-M)$ at the age of 12 years.

\begin{tabular}{|c|c|c|c|c|c|c|}
\hline & \multicolumn{6}{|c|}{ PA girls } \\
\hline & $\mathrm{PA}+\mathrm{PP}, n=20$ & $\mathrm{PA}-\mathrm{PP}, n=16$ & $P$ & $\mathrm{PA}+\mathrm{M}, n=13$ & $\mathrm{PA}-\mathrm{M}, n=23$ & $P$ \\
\hline \multicolumn{7}{|l|}{ At birth } \\
\hline Gestational age, weeks & 40.2 (38.9 to 41.0$)$ & 40.4 (39.0 to 41.0$)$ & $0.949^{a}$ & 40.3 (38.9 to 41.0$)$ & 40.4 (39.2 to 41.4$)$ & $0.882^{\mathrm{a}}$ \\
\hline Birth weight, kg & 3.44 (3.01 to 3.72 ) & 3.52 (3.13 to 3.80$)$ & $0.787^{\mathrm{a}}$ & 3.41 (3.11 to 3.57 ) & 3.63 (3.19 to 4.01 ) & $0.122^{\mathrm{a}}$ \\
\hline Birth weight SDS & $-0.16(-0.75$ to 0.43$)$ & $-0.07(-0.63$ to 0.49$)$ & 0.808 & $-0.36(-0.71$ to -0.01$)$ & $0.30(-0.62$ to 1.23$)$ & 0.097 \\
\hline Birth length (BL), cm & 49.8 (48.0 to 50.8) & 50.0 (48.3 to 51.0) & $0.498^{a}$ & 49.0 (48.0 to 50.0) & $51.0(49.5$ to 51.8$)$ & $0.028^{a}$ \\
\hline BL SDS & $-0.15(-0.62$ to 0.32$)$ & $-0.06(-0.63$ to 0.52$)$ & 0.785 & $-0.45(-0.77$ to -0.13$)$ & $0.50(-0.22$ to 1.22$)$ & 0.005 \\
\hline \multicolumn{7}{|l|}{ At 7 years of age } \\
\hline Height SDS & 1.34 (0.78 to 1.90$)$ & 0.61 (0.14 to 1.09$)$ & 0.051 & $1.23(0.80$ to 1.66$)$ & $0.63(-0.14$ to 1.40$)$ & 0.125 \\
\hline BMI SDS & $1.23(0.74$ to 1.72$)$ & $0.81(0.14$ to 1.47$)$ & 0.274 & $1.20(0.75$ to 1.65$)$ & $0.77(-0.02$ to 1.57$)$ & 0.289 \\
\hline DHEAS, $\mu \mathrm{mol} / \mathrm{L}$ & $2.5(1.7$ to 3.6$)$ & $1.8(1.4$ to 2.2$)$ & $0.037^{a}$ & $2.1(1.6$ to 3.1$)$ & $2.1(1.4$ to 2.5$)$ & $0.830^{a}$ \\
\hline Androstenedione, $\mathrm{nmol} / \mathrm{L}$ & 3.3 (2.7 to 4.4$)$ & $2.4(1.3$ to 3.4$)$ & $0.037^{a}$ & 3.1 (2.7 to 4.0$)$ & $3.2(1.6$ to 4.4$)$ & $0.564^{a}$ \\
\hline Insulin, mU/L & $6.0(5.5$ to 7.8$)$ & 4.5 (3.1 to 7.4$)$ & $0.161^{\mathrm{a}}$ & $6.1(4.7$ to 7.4$)$ & $4.5(2.7$ to 7.1$)$ & $0.121^{a}$ \\
\hline IGF-1, nmol/L & $26.0(22.2$ to 31.0$)$ & $21.0(17.3$ to 26.0$)$ & $0.037^{a}$ & 26.0 (21.8 to 33.8$)$ & 20.0 (17.5 to 24.0$)$ & $0.009^{a}$ \\
\hline \multicolumn{7}{|l|}{ At 12 years of age } \\
\hline Height, cm & 162.6 (158.9 to 166.2$)$ & $156.9(152.5$ to 161.3$)$ & 0.032 & 162.5 (159.7 to 165.2$)$ & $155.8(149.8$ to 161.7$)$ & 0.036 \\
\hline Height SDS & $1.25(0.73$ to 1.76$)$ & $0.44(-0.18$ to 1.07$)$ & 0.041 & $1.23(0.84$ to 1.63$)$ & $0.28(-0.55$ to 1.11$)$ & 0.039 \\
\hline Weight, kg & 55.5 (46.2 to 66.6$)$ & 49.9 (39.5 to 70.2$)$ & $0.301^{\mathrm{a}}$ & 55.6 (48.0 to 67.4$)$ & 45.7 (38.3 to 68.9) & $0.078^{a}$ \\
\hline $\mathrm{BMI}, \mathrm{kg} / \mathrm{m}^{2}$ & 20.8 (18.8 to 25.3 ) & 20.1 (17.5 to 26.5$)$ & $0.702^{\mathrm{a}}$ & 21.4 (19.0 to 25.4$)$ & 18.1 (17.6 to 26.1$)$ & $0.249^{a}$ \\
\hline BMI SDS & 0.87 (0.47 to 1.27$)$ & 0.74 (0.16 to 1.32$)$ & 0.692 & 0.96 (0.58 to 1.34$)$ & $0.56(-0.08$ to 1.20$)$ & 0.227 \\
\hline $\mathrm{cMPH}, \mathrm{cm}$ & 167.0 (164.2 to 169.7$)$ & 166.9 (164.6 to 169.2$)$ & 0.987 & 166.8 (164.6 to 169.1$)$ & $167.2(163.9$ to 170.5$)$ & 0.842 \\
\hline Maternal age at menarche, years & $12.9(11.1$ to 14.0$)$ & $12.0(11.3$ to 13.8$)$ & $0.759^{\mathrm{a}}$ & $12.0(11.0$ to 13.0$)$ & $13.0(12.3$ to 14.0$)$ & $0.012^{a}$ \\
\hline Early paternal pubertal growth spurt, yes \% & 30 & 13 & $0.209^{b}$ & 26 & 15 & $0.458^{b}$ \\
\hline
\end{tabular}

Values are expressed as mean (95\% confidence interval), and statistical differences between the study groups

are analyzed using independent samples t-test, unless noted otherwise. Significant p-values $(<0.05)$ are highlighted with bold.

${ }^{a}$ Median (interquartile range) and Mann-Whitney $U$ test for non-normally distributed variables.

${ }^{b}$ Percentage and Pearson $\chi^{2}$ test for categorical variable.

$P A$, premature adrenarche; PP, premature pubarche; $M$, menarche; SDS, standard deviation score;

$B M I$, body mass index; DHEAS, dehydroepiandrosterone sulfate; IGF-1, insulin-like growth factor 1; cMPH, corrected mid-parental height.

(Tanner $\mathrm{B} \geq 2$ or testicular volume $\geq 4 \mathrm{~mL}$ ) between the PA and control children; $95.4 \%$ of the participating girls and $55.6 \%$ of the boys were pubertal. However, in a more detailed analysis of pubertal development, the PA girls were more advanced than the controls as they had more often reached menarche (63.9 vs $25.0 \%$, $P<0.001$; Table 3), had higher pubic hair stage $(P<0.001$; Figure 1), and a trend for more advanced breast development $(P=0.072$; Figure 1). When analyzing the PA girls, the PA + PP girls had higher percentage of reached menarche compared with that of the PA - PP girls ( 80.0 vs $43.8 \%, P=0.024$; Table 3 ). Among the boys, no statistical difference in pubertal development was found between the PA and control groups.

\section{Associations with Menarcheal Timing}

Logistic regression analyses on determinants of menarche for all girls is shown in Table 4. In univariate analyses, lower BL SDS, the history of PA, earlier maternal menarche, higher prepubertal BMI, serum IGF-1, and DHEAS concentrations were all significant determinants of earlier menarche (data not shown). In multivariate analyses, lower BL SDS, lower maternal age at menarche, and a higher prepubertal IGF-1 concentration were independently associated with earlier menarche. In subgroup analyses comparing the PA girls with and without menarche, the postmenarcheal PA girls had lower BL, higher prepubertal IGF-1 concentrations, and taller stature at the age of 12 years (Table 2).

\section{Comparison of Parental Heights and Pubertal Timing between the Study Groups}

The history of parental pubertal development and cMPH are depicted in Tables 1 and 2. cMPH had a trend to be higher in the PA girls compared with the control ones $(166.9$ vs $165.0 \mathrm{~cm}$, $P=0.064)$. Maternal age at menarche did not differ between the PA and control girls. However, maternal median age at menarche was lower in the PA girls with menarche compared with those without menarche (12.0 vs 13.0 years, $P=0.012$ ). Percentages of the fathers with early pubertal growth spurt was higher in the PA girls compared with the control girls (22 vs $6 \%, P=0.024$ ). No differences were found in $\mathrm{CMPH}$, maternal age at menarche, or the percentage of the fathers with early pubertal growth spurt between the PA and control boys or the PA + PP and PA - PP girls (Tables 1 and 2).

\section{DISCUSSION}

In this follow-up study, we examined mostly full-term and appropriate for gestational age (AGA)-born children with a history of PA and their healthy controls at the age of 12 years. In prepuberty, the PA girls had presented with advanced statural growth, higher weight-for-height, and higher serum IGF-1 concentrations than the control children (14). Most of these children (61.6\%) 
A Tanner stage in $\mathrm{B} / \mathrm{G}$ scale $\quad 1 \quad \square 2 \quad \square 3 \quad \square 4 \quad \square 5$

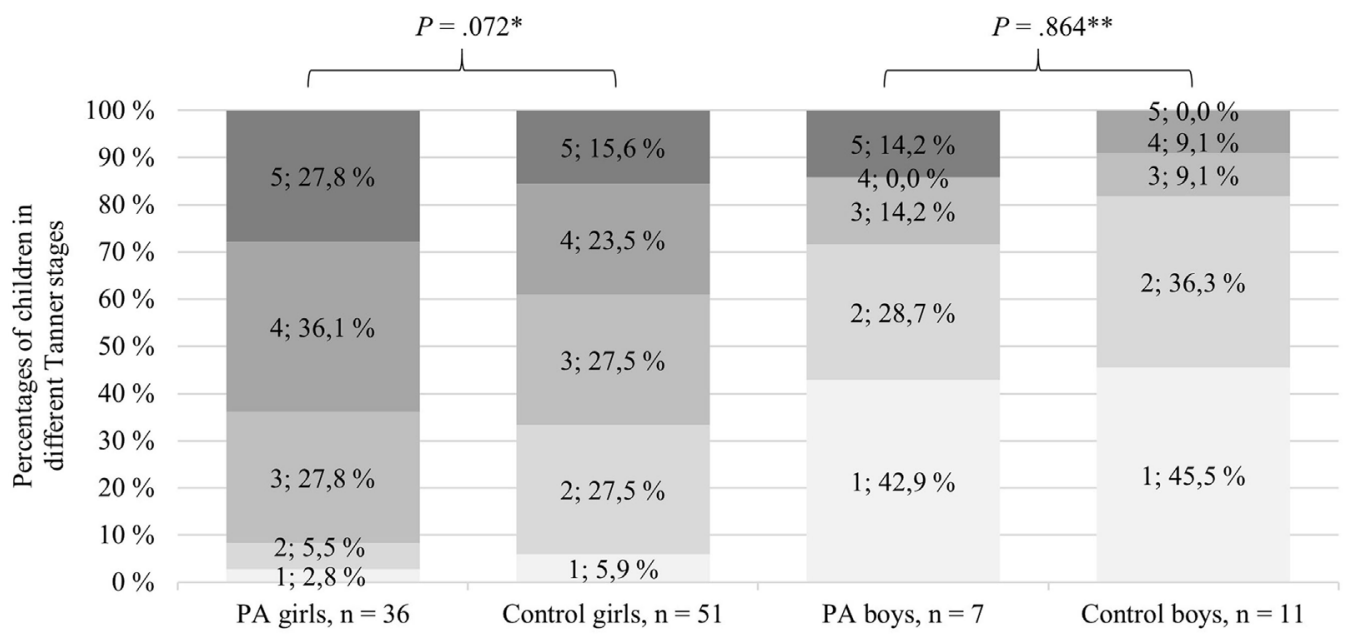

B Tanner stage in P scale $\quad 1 \quad 2 \quad \square=3 \quad \square \quad \square 5$

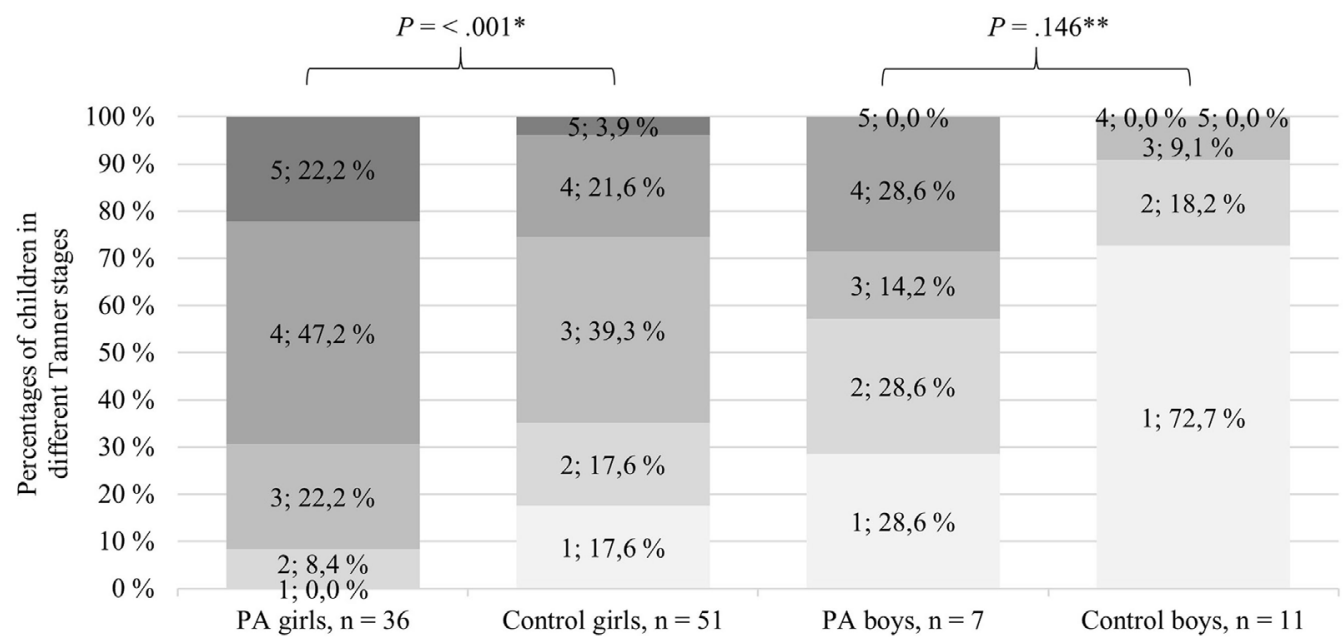

FIGURE 1 | Pubertal development in Tanner B/G (A) and P scale (B) for children with premature adrenarche (PA) and controls at the age of 12 years. Gray shades represent Tanner stage scores, and darker shades reflect more advanced pubertal development. *Pearson $\chi^{2}$ test or ${ }^{* *}$ Fisher's exact test was used to analyze statistical differences between the PA and control girls/boys, respectively.

TABLE 3 | Pubertal development of children with PA and control children at the age of 12 years, including subgroup analyses between the PA girls with (PA + PP) and without premature pubarche (PA - PP).

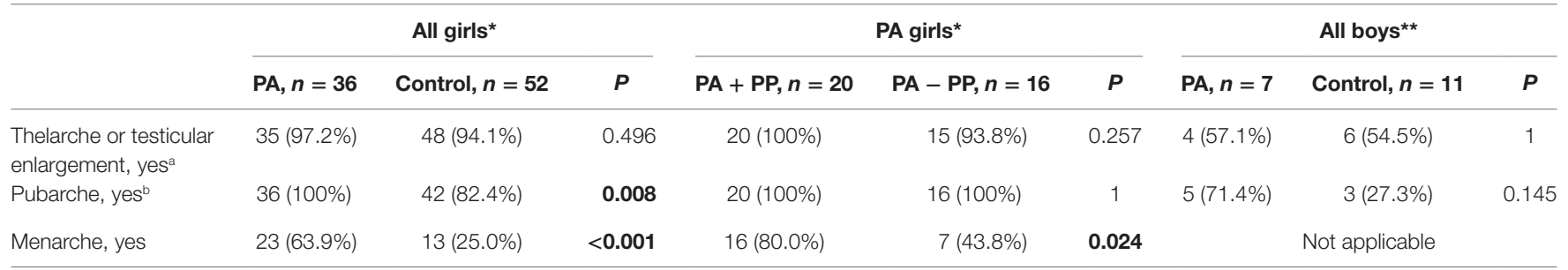

Values are presented as $n(\%)$, and statistical differences between the study groups were analyzed using either ${ }^{*} P$ earson $\chi^{2}$ test or ${ }^{* *}$ Fisher's exact test. Significant $p$-values (<0.05) are highlighted with bold.

Tanner $B \geq 2$ or testicular volume $\geq 4 \mathrm{~mL}$.

${ }^{b}$ Tanner $P \geq 2$.

Both ${ }^{a}$ and ${ }^{b} n=51$ for control girls.

$P A$, premature adrenarche; $P P$, premature pubarche. 
TABLE 4 | Logistic regression models predicting menarche by the age of 12 years.

\section{Regression $P \quad$ Odds ratio $(95 \% \mathrm{Cl})^{\mathrm{a}}$ coefficient $^{\mathrm{a}}$}

\section{Model 1}

Age, years ${ }^{b}$

Birth length SDS

Birth weight SDS

PA

Prepubertal BMI SDS

Maternal age at menarche, years

Early paternal pubertal growth

spurt ${ }^{\circ}$

Model 2

Age, years

DHEAS SDS

Androstenedione SDS

Insulin SDS

IGF-1 SDS

$-2.16$

0.292

0.002

0.286

0.083

1.21

0.66

0.052

$\mathbf{0 . 0 0 1}$

0.302

1.10

$1.66(0.65-4.25)$

$0.12(0.03-0.46)$

$1.81(0.61-5.35)$

$3.37(0.85-13.3)$

$1.94(1.00-3.78)$

$0.30(0.15-0.62)$

$2.99(0.37-23.9)$

$\begin{array}{lll}0.14 & 0.718 & 1.14(0.55-2.38) \\ 0.20 & 0.625 & 1.22(0.55-2.70) \\ 0.32 & 0.478 & 1.38(0.57-3.35) \\ 0.30 & 0.291 & 1.35(0.77-2.35) \\ 0.76 & \mathbf{0 . 0 1 3} & 2.14(1.18-3.88)\end{array}$

Dependent variable: menarche at the age of 12 years, yes or no.

Estimated size of the effects of all variables in the models: Model 1, $R^{2}=0.65$;

Model 2, $R^{2}=0.30$. Significant $p$-values $(<0.05)$ are highlighted with bold.

${ }^{a}$ Regression coefficients and odds ratios are

adjusted for all other variables in the models.

${ }^{b}$ Age at the initial evaluation visit in prepuberty.

'Paternal pubertal growth spurt over 1 year earlier compared with their peers.

$\mathrm{Cl}$, confidence interval; SDS, standard deviation score; $P A$, premature adrenarche;

$B M I$, body mass index; DHEAS, dehydroepiandrosterone sulfate; IGF-1, insulin-like

growth factor 1 .

participated in this follow-up visit and, compared with healthy peers, the PA girls were still taller and heavier, and had more advanced pubertal development. Moreover, those PA girls who had presented with premature pubarche $(\mathrm{PP})$ at the initial visit in prepuberty were taller and had higher percentage of reached menarche at the age of 12 years than the PA girls without PP. The number of boys in this study was too small to detect any statistically significant differences in anthropometry or pubertal development. In logistic regression analyses, earlier maternal menarche, lower BL SDS, and higher serum IGF-1 concentrations were significant explanatory factors for earlier menarche in all girls of this study. The PA girls with menarche had lower BL SDS, higher prepubertal IGF-1 concentrations and taller stature at the age of 12 years than the PA girls without menarche.

To date, there are only two published reports investigating PA children in a prospective setting $(9,18)$. In both of these, contrary to our study, there was no control group, many participants were born SGA, and all children with PA had PP. In one of these studies, 187 girls with PP were followed from diagnosis to early adulthood: the PP children had earlier menarche than the girls in the general population (18). In the other more recent longitudinal study, 52 girls with PP were followed from the diagnosis of PP (9). Approximately one-third of these girls were obese, which was more than in the reference population. Both thelarche and menarche occurred earlier than in the general population being still within the normal range. One-third (30.8\%) of these children had reached final height, with no difference to the normal population standards.

Some cross-sectional studies have explored pubertal development in children with a history of PA/PP. In a Finnish study investigating growth and maturation of 23 teenaged mostly AGA-born PA girls, menarche occurred 0.5 years earlier than in the general female population (13). Evidence for earlier pubertal timing in PA children was also reported in two other retrospective studies on 85 AGA-born Israeli and 37 multiethnic PA girls: menarche occurred earlier in PA girls than controls (26) or reference population (27), and thelarche occurred earlier compared with the control group (27). In contrast, some authors did not find any difference in pubertal timing in PA girls compared with general population (12). Our present findings are well in line with these previous longitudinal and retrospective studies showing slightly earlier pubertal development after PA.

There are also some retrospective or cross-sectional studies on pubertal growth and body composition in subjects with the history of PA. In one study, non-obese postmenarcheal adolescent girls with the history of PP had higher total body fat mass and more unfavorable fat distribution than those without a history of PP (28). In two other studies, PA children were taller than the general population at early puberty $(12,13)$. Our study, however, is the first one comparing pubertal height between PA/PP children and carefully matched controls in a follow-up setting, and confirms that PA girls remain taller still at pubertal age.

In the light of current data, PA has an impact on pubertal timing, but the mechanisms behind this phenomenon are not clear. First, excess androgen exposure in PA could lead to increased peripheral conversion of weak adrenal androgens to more potent androgens and estrogens. In a recent longitudinal study on 252 healthy peripubertal girls, serum DHEAS, androstenedione and estrone concentrations rose before thelarche and the increase in serum estradiol concentration (29). The authors did not study the age of menarche in these girls, but suggested that peripheral conversion of adrenal androgens might lead to breast development without gonadotropin activation and might thus slow the tempo from thelarche to menarche. This study setting was different, but our results do not support this hypothesis as menarche was earlier without a significant difference of breast development in the PA girls compared with the controls. Second, adrenal androgens may accelerate gonadotrophin-dependent pubertal development. DHEAS, the most prominent biomarker in PA, is known to act as a neurosteroid (30), and some authors have speculated that adrenal androgens might have some effects on pubertal onset and tempo via $\gamma$-aminobutyric acid inhibition to the gonadotropin-releasing hormone pulse generator (31). Third, the most probable connection between adrenal and gonadal maturation is insulin-IGF-1 axis, as increased concentrations of IGF-1 and insulin have been associated with both PA $(6,14,16,17)$ and central precocious puberty (32).

Advanced early childhood growth (usually associated with PA) tends to induce greater weight gain, taller stature, and hyperinsulinism in later childhood (33). Hyperinsulinism may contribute to hepatic IGF-1 formation leading to increased IGF- 1 concentrations. IGF-1, in turn, may be a key factor in the postnatal adrenal maturation (34) and adrenarche in girls (35). Interestingly, rapid weight gain and growth in early childhood (reflecting good nutritional environment) may induce a 
childhood endocrine milieu where hyperinsulinism, elevated IGF-1, enhanced adrenal androgen secretion, increased peripheral metabolism of these weak androgens, and lower SHBG concentrations (leading to increased free androgen concentrations) could all promote pulsatile gonadotropin-releasing hormone activity (33). Moreover, some hypothesized etiological factors of PA, including low birth weight $(5,10,36)$ and higher animal protein intake in early childhood (37), may promote growth acceleration and earlier pubertal development independently of PA $(19,31)$. In our own cohort, mostly AGA-born PA girls had early growth acceleration, hyperinsulinism, elevated IGF-1, and decreased SHBG concentrations $(14,20)$, and as our current study indicates, also earlier pubertal development. Moreover, the PA girls with PP were taller and had more often reached menarche by the age of 12 years than the PA girls without PP indicating a "more severe" phenotype. In addition, earlier menarcheal timing was associated with lower BL SDS and higher prepubertal IGF-1 concentration in all girls of our cohort, and the PA girls with menarche had lower BL SDS, higher prepubertal IGF-1 concentrations, and taller stature at the age of 12 years than the PA girls without menarche. Our results support the hypothesis that early factors in the life of PA girls may contribute to the acceleration of growth and maturation.

Our present study is the first longitudinal study investigating pubertal development and growth of PA children including a healthy control group matched for age and sex. All children were examined at the age of 12 years by one single trained physician, which increases the reliability of the clinical assessment. However, our study has limitations. The age of 12 years does not allow complete evaluation of later pubertal development. The cohort size was relatively small, and $38.4 \%$ of the original cohort did not attend the current follow-up visit. Especially for the boys, the number of participants was too small for any strong conclusions. It should be noted that mean $\mathrm{CMPH}$ and the percentage of the fathers with early pubertal growth spurt were higher in the PA girls compared with the controls, and maternal menarche was earlier in the PA girls with menarche compared with the PA girls without menarche. Thus, the genetic background may partly explain our observed differences in height and age of menarche between the study groups. Unfortunately, we do not know if the parents themselves have had PA.

In conclusion, the PA girls remained taller and more overweight than the control girls at the age of 12 years, and they

\section{REFERENCES}

1. Utriainen P, Laakso S, Liimatta J, Jääskeläinen J, Voutilainen R. Premature adrenarche - a common condition with variable presentation. Horm Res Paediatr (2015) 83:221-31. doi:10.1159/000369458

2. Voutilainen R, Jääskeläinen J. Premature adrenarche: etiology, clinical findings, and consequences. J Steroid Biochem Mol Biol (2015) 145:226-36. doi:10.1016/j.jsbmb.2014.06.004

3. Mäntyselkä A, Jääskeläinen J, Lindi V, Viitasalo A, Tompuri T, Voutilainen R, et al. The presentation of adrenarche is sexually dimorphic and modified by body adiposity. J Clin Endocrinol Metab (2014) 99:3889-94. doi:10.1210/ jc.2014-2049

4. Paterson WF, Ahmed SF, Bath L, Donaldson MD, Fleming R, Greene SA, et al. Exaggerated adrenarche in a cohort of Scottish children: clinical features presented with earlier menarche. In addition, the PA girls with PP were taller and had earlier menarche at the age of 12 years than the PA girls without PP. The factors predicting earlier menarche in all girls included lower BL and higher prepubertal serum IGF-1 concentration which were stronger determinants than PA or prepubertal concentrations of adrenal androgens. Further longitudinal studies are needed to verify pubertal timing and other long-term consequences of PA.

\section{ETHICS STATEMENT}

This study was carried out in accordance with the Declaration of Helsinki. Informed consent was obtained from all participating children and their parents. The protocol was approved by the Research Ethics Committee of the Hospital District of Northern Savo.

\section{AUTHOR CONTRIBUTIONS}

JJ, RV, and PU conceived the project and designed the study; PU and JL performed collection and handling of the data; JL and JJ analyzed the data; all the authors discussed the data and accepted the final draft; JL wrote the manuscript with contributions from all the authors.

\section{ACKNOWLEDGMENTS}

The authors are grateful to all children and their families participating in this study and thank Ms. Leila Antikainen for biochemical analyses.

\section{FUNDING}

This work was supported by Kuopio University Hospital (Kuopio, Finland), the Foundation for Pediatric Research (Helsinki, Finland), the Finnish Medical Foundation (Helsinki, Finland), the Päivikki and Sakari Sohlberg Foundation (Helsinki, Finland), and the Sigrid Jusélius Foundation (Helsinki, Finland). The funders had no role in: (1) study design; (2) the collection, analysis, and interpretation of data; (3) the writing of the report; and (4) the decision to submit the paper for publication. and biochemistry. Clin Endocrinol (Oxf) (2010) 72:496-501. doi:10.1111/ j.1365-2265.2009.03739.x

5. Neville KA, Walker JL. Precocious pubarche is associated with SGA, prematurity, weight gain, and obesity. Arch Dis Child (2005) 90:258-61. doi:10.1136/ adc.2004.053959

6. Utriainen P, Jääskeläinen J, Romppanen J, Voutilainen R. Childhood metabolic syndrome and its components in premature adrenarche. JClin Endocrinol Metab (2007) 92:4282-5. doi:10.1210/jc.2006-2412

7. Denburg MR, Silfen ME, Manibo AM, Chin D, Levine LS, Ferin M, et al. Insulin sensitivity and the insulin-like growth factor system in prepubertal boys with premature adrenarche. J Clin Endocrinol Metab (2002) 87:5604-9. doi:10.1210/jc.2002-020896

8. Vuguin P, Linder B, Rosenfeld RG, Saenger P, DiMartino-Nardi J. The roles of insulin sensitivity, insulin-like growth factor I (IGF-I), and IGF-binding 
protein-1 and -3 in the hyperandrogenism of African-American and Caribbean Hispanic girls with premature adrenarche. J Clin Endocrinol Metab (1999) 84:2037-42. doi:10.1210/jcem.84.6.5722

9. DeFerran K, Paiva IA, Garcia LdS, Gama MdP, Guimarães MM. Isolated premature pubarche: report of anthropometric and metabolic profile of a Brazilian cohort of girls. Horm Res Paediatr (2011) 75:367-73. doi:10.1159/ 000324107

10. Ibañez L, Potau N, Francois I, de Zegher F. Precocious pubarche, hyperinsulinism, and ovarian hyperandrogenism in girls: relation to reduced fetal growth. J Clin Endocrinol Metab (1998) 83:3558-62. doi:10.1210/jc.83.10.3558

11. Voutilainen R, Perheentupa J, Apter D. Benign premature adrenarche: clinical features and serum steroid levels. Acta Paediatr Scand (1983) 72:707-11. doi:10.1111/j.1651-2227.1983.tb09798.x

12. Ibañez L, Virdis R, Potau N, Zampolli M, Ghizzoni L, Albisu MA, et al. Natural history of premature pubarche: an auxological study. J Clin Endocrinol Metab (1992) 74:254-7. doi:10.1210/jc.74.2.254

13. Pere A, Perheentupa J, Peter M, Voutilainen R. Follow up of growth and steroids in premature adrenarche. Eur J Pediatr (1995) 154:346-52. doi:10.1007/ BF02072100

14. Utriainen $P$, Voutilainen R, Jääskeläinen J. Girls with premature adrenarche have accelerated early childhood growth. J Pediatr (2009) 154:882-7. doi:10.1016/j. jpeds.2008.12.038

15. Güven A, Cinaz P, Ayvali E. Are growth factors and leptin involved in the pathogenesis of premature adrenarche in girls? J Pediatr Endocrinol Metab (2005) 18:785-91. doi:10.1515/JPEM.2005.18.8.785

16. Silfen ME, Manibo AM, Ferin M, McMahon DJ, Levine LS, Oberfield SE. Elevated free IGF-I levels in prepubertal Hispanic girls with premature adrenarche: relationship with hyperandrogenism and insulin sensitivity. JClin Endocrinol Metab (2002) 87:398-403. doi:10.1210/jcem.87.1.8143

17. Sopher AB, Jean AM, Zwany SK, Winston DM, Pomeranz CB, Bell JJ, et al. Bone age advancement in prepubertal children with obesity and premature adrenarche: possible potentiating factors. Obesity (Silver Spring) (2011) 19:1259-64. doi:10.1038/oby.2010.305

18. Ibañez L, Jimenez R, de Zegher F. Early puberty-menarche after precocious pubarche: relation to prenatal growth. Pediatrics (2006) 117:117-21. doi:10.1542/peds.2005-0664

19. Hokken-Koelega AC. Timing of puberty and fetal growth. Best Pract Res Clin Endocrinol Metab (2002) 16:65-71. doi:10.1053/beem.2002.0181

20. Utriainen P, Voutilainen R, Jääskeläinen J. Continuum of phenotypes and sympathoadrenal function in premature adrenarche. Eur J Endocrinol (2009) 160:657-65. doi:10.1530/EJE-08-0367

21. Saari A, Sankilampi U, Hannila ML, Kiviniemi V, Kesseli K, Dunkel L. New Finnish growth references for children and adolescents aged 0 to 20 years: length/height-for-age, weight-for-length/height, and body mass indexfor-age. Ann Med (2011) 43:235-48. doi:10.3109/07853890.2010.515603

22. Sankilampi U, Hannila ML, Saari A, Gissler M, Dunkel L. New population-based references for birth weight, length, and head circumference in singletons and twins from 23 to 43 gestation weeks. Ann Med (2013) 45:446-54. doi:10.3109/07853890.2013.803739

23. Marshall WA, Tanner JM. Variations in pattern of pubertal changes in girls. Arch Dis Child (1969) 44:291-303. doi:10.1136/adc.44.235.291

24. Marshall WA, Tanner JM. Variations in the pattern of pubertal changes in boys. Arch Dis Child (1970) 45:13-23. doi:10.1136/adc.45.239.13
25. Tanner JM, Goldstein H, Whitehouse RH. Standards for children's height at ages 2-9 years allowing for height of parents. Arch Dis Child (1970) 45:755-62. doi:10.1136/adc.45.244.755

26. Oron T, Lebenthal Y, de Vries L, Yackobovitch-Gavan M, Phillip M, Lazar L. Interrelationship of extent of precocious adrenarche in appropriate for gestational age girls with clinical outcome. J Pediatr (2012) 160:308-13. doi:10.1016/j.jpeds.2011.08.009

27. Miller D, Emans SJ, Kohane I. Follow-up study of adolescent girls with a history of premature pubarche. JAdolesc Health (1996) 18:301-5 doi:10.1016/1054-139X(95)00234-J

28. Ibañez L, Ong K, de Zegher F, Marcos MV, del Rio L, Dunger DB. Fat distribution in non-obese girls with and without precocious pubarche: central adiposity related to insulinaemia and androgenaemia from prepuberty to postmenarche. Clin Endocrinol (2003) 58:372-9. doi:10.1046/j.1365-2265.2003.01728.x

29. Biro FM, Pinney SM, Huang B, Baker ER, Walt Chandler D, Dorn LD. Hormone changes in peripubertal girls. JClin Endocrinol Metab (2014) 99:3829-35. doi:10.1210/jc.2013-4528

30. Campbell B. Adrenarche and the evolution of human life history. Am J Hum Biol (2006) 18:569-89. doi:10.1002/ajhb.20528

31. Remer T, Shi L, Buyken AE, Maser-Gluth C, Hartmann MF, Wudy SA. Prepubertal adrenarchal androgens and animal protein intake independently and differentially influence pubertal timing. J Clin Endocrinol Metab (2010) 95:3002-9. doi:10.1210/jc.2009-2583

32. Sorensen K, Aksglaede L, Petersen JH, Andersson AM, Juul A. Serum IGF1 and insulin levels in girls with normal and precocious puberty. Eur J Endocrinol (2012) 166:903-10. doi:10.1530/EJE-12-0106

33. Dunger DB, Ahmed ML, Ong KK. Early and late weight gain and the timing of puberty. Mol Cell Endocrinol (2006) 254-255:140-5. doi:10.1016/j.mce.2006.04.003

34. Baquedano MS, Berensztein E, Saraco N, Dorn GV, de Davila MT, Rivarola MA, et al. Expression of the IGF system in human adrenal tissues from early infancy to late puberty: implications for the development of adrenarche. Pediatr Res (2005) 58:451-8. doi:10.1203/01.PDR.0000179392.59060.93

35. Guercio G, Rivarola MA, Chaler E, Maceiras M, Belgorosky A. Relationship between the growth hormone/insulin-like growth factor-I axis, insulin sensitivity, and adrenal androgens in normal prepubertal and pubertal girls. J Clin Endocrinol Metab (2003) 88:1389-93. doi:10.1210/jc.2002-020979

36. Charkaluk ML, Trivin C, Brauner R. Premature pubarche as an indicator of how body weight influences the onset of adrenarche. Eur J Pediatr (2004) 163:89-93. doi:10.1007/s00431-003-1358-9

37. Shi L, Wudy SA, Buyken AE, Hartmann MF, Remer T. Body fat and animal protein intakes are associated with adrenal androgen secretion in children. Am J Clin Nutr (2009) 90:1321-8. doi:10.3945/ajcn.2009.27964

Conflict of Interest Statement: The authors declare that the research was conducted in the absence of any commercial or financial relationships that could be construed as a potential conflict of interest.

Copyright (c) 2017 Liimatta, Utriainen, Voutilainen and Jä̈skeläinen. This is an open-access article distributed under the terms of the Creative Commons Attribution License (CC BY). The use, distribution or reproduction in other forums is permitted, provided the original author(s) or licensor are credited and that the original publication in this journal is cited, in accordance with accepted academic practice. No use, distribution or reproduction is permitted which does not comply with these terms. 